Highly Energetic Physical Processes and

Mechanisms for Emission from Astrophysical Plasmas

IAU Symposium, Vol. 195, 2000

P. C. H. Martens, S. Tsuruta, and M. A. Weber, eds.

\title{
Multiwavelength Diagnostics of Pulsar Plasmas
}

\author{
R. W. Romani \\ Dept. of Physics, Stanford University, CA 94305-4060, U.S.A.
}

\begin{abstract}
The phase-resolved broadband (optical to gamma-ray) spectral energy distributions of spin-powered pulsars contain important diagnostics of the emission zone plasma and its acceleration. I summarize some recent observations of young pulsars, describe models for the various spectral components, and argue that the next generation of telescopes and spacecraft will allow a true mapping of the $\mathrm{e}^{ \pm}$plasma flow in the pulsar magnetosphere. Of course, spectral lines and breaks are more powerful tracers than the spectral energy distribution, and since we believe that synchrotron emission dominates in the near-IR to UV, features are expected that will allow even more detailed probes of the plasma physics. Most young pulsars are, however, quite faint, and new technology is needed to extract these diagnostics.
\end{abstract}

\section{Introduction - Multiwavelength Pulses}

There has been much recent progress in detecting and understanding high-energy $\left(\sim 10^{0}-10^{7} \mathrm{eV}\right)$ magnetospheric emission from spin-powered pulsars. We must distinguish this emission from the coherent radio pulsations which provide the primary channel for pulsar identification and dynamics/evolutionary studies. The mechanism of the radio emission is very difficult to interpret (but see Lyutikov, Blandford, \& Machabeli 2000, these Proceedings). Similarly, we separate the thermal flux from the surface atmosphere (reviewed by Pavlov \& Zavlin 2000, these Proceedings), although this emission provides important boundary conditions on the high-energy emitting gaps. Finally, we do not review the important data obtained on the magnetosphere generated particles and wind that may be extracted from the surrounding plerionic flux (e.g., see Becker 2000; Pacini 2000, both in these Proceedings).

Thus, we focus on the nonthermal magnetospheric emission. This is highly pulsed and, hence, highly beamed. With pulse profiles having characteristic component widths $<0.1$ in phase and the cusp of the Crab optical pulse extending over no more than $\delta \phi \sim 0.01$, we must have radiation dominated by $e^{ \pm}$ with $\Gamma \geq 10-100$. We also must have relatively narrow radiating zones in the magnetosphere, where, again, the component widths restrict us to a few percent of the field line volume. This is an especially challenging restriction when one recalls that for some intermediate-age pulsars, e.g., PSR B1055-52 and Geminga, the apparent photon luminosity is $10 \%$ or more of the spin-down power. This emphasizes the fact that pulsar emission is unlike the vast majority of problems 


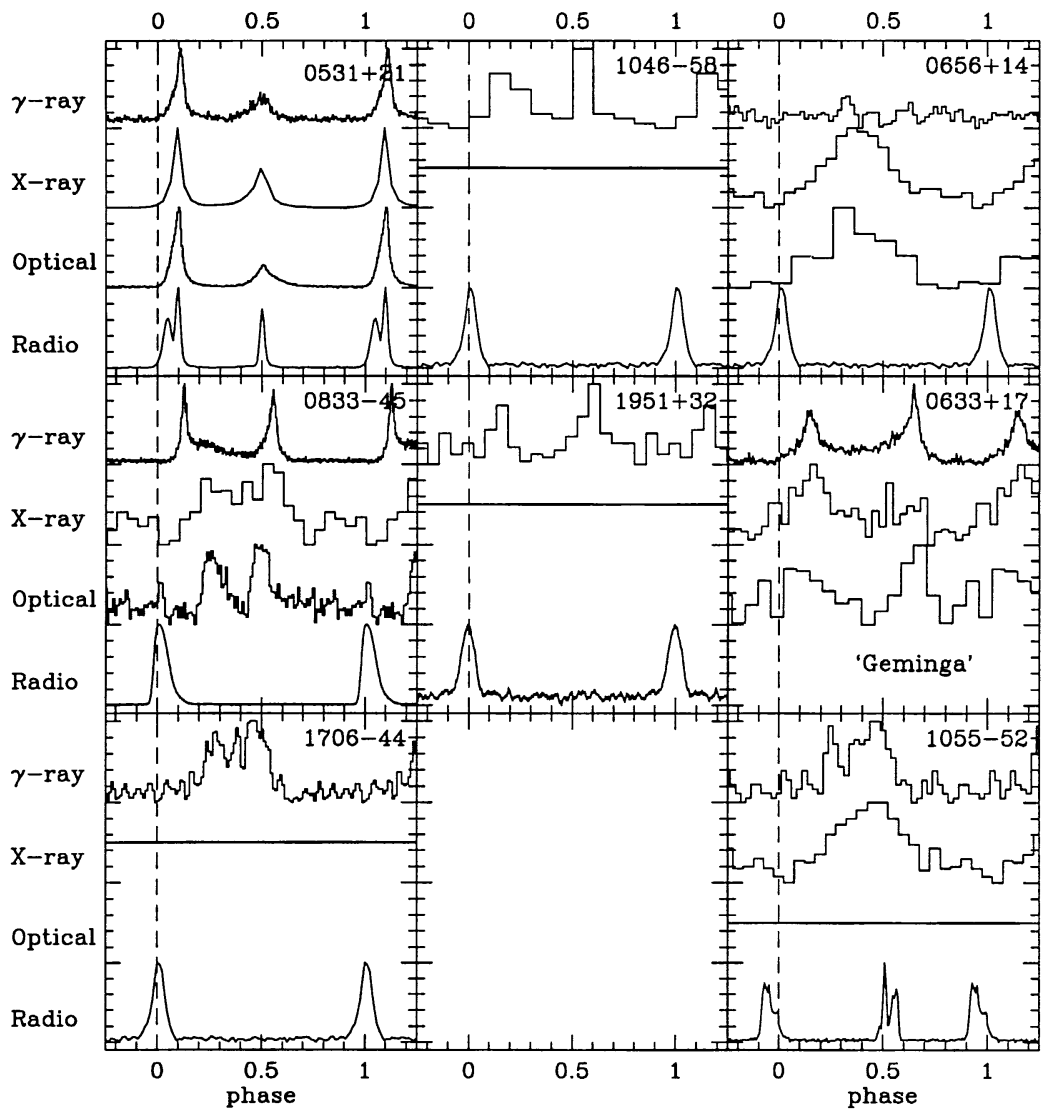

Figure 1. Young, $\tau<10^{6} \mathrm{y}$ pulsars detected in $\gamma$-rays. Light curves from the literature, phased to the best estimate of magnetic axis. The axis phase is determined from polarization sweep structure and radio pulse modeling, when possible. Geminga's phase is set from outer gap fits to the $\mathrm{GeV}$ profile. The uncertainty of phasing between energy bands is set by clock inaccuracies (e.g., ROSAT) and DM uncertainty in the radio. Radio light curves are generic for PSRs 1046 and 0656, and unpulsed detections are indicated by horizontal lines.

in astrophysics, where our models grow from 1-D single zone sums with isotropic particle populations and $1-\mathrm{D}$ radiation flows.

So, the first step in interpreting pulsar beaming comes from inspection of the light curves across a broad energy range. In Figure 1, we show a new compilation of light curves for the young $\left(\tau<10^{6} \mathrm{y}\right)$ pulsars that are either detected or are strong candidates in high-energy $\gamma$-rays. These have been assembled from the literature using the best estimates of the phasing to the radio emission. To highlight the common features, these profiles has been plotted at a common absolute phase, so that the magnetic axis sweeps closest to the line of sight at $\phi=0$; this is generally marked by the radio pulse. A few cases are special: 
for Crab, the magnetic axis leads the precursor slightly as fit from the optical polarization sweep in an outer gap "Radhakrishnan \& Cooke "-type solution (1969; Romani \& Yadigaroglu 1995); for PSR B1055-52, the radio polarization and frequency dependence suggest partial cone emission from an interpulsar (Lyne \& Manchster 1988); and for Geminga, since no firm radio detection has been made, the axis shown is that determined from the $\mathrm{GeV}$ light curve in an outer magnetosphere gap model (Romani \& Yadigaroglu 1995, hereafter RY95).

In the outer magnetosphere picture (e.g., Morini 1983), the light curves displayed in this way make a fairly coherent set. The $\mathrm{GeV}$ light curves have double peaks which come from the pole at $\phi=0.5$ and are beamed towards the spin equator $(\zeta=\pi / 2)$. Aberration shifts the peaks forward in phase and pulsars viewed at small $\zeta$ have a smaller $\mathrm{GeV}$ peak separation; the model provides a quantitative match to the observed profiles (RY95). The nonthermal optical and hard X-ray emissions lie largely within the $\gamma$-ray peaks and arise at lower altitudes, close to the null charge surface that serves as the inner boundary of the acceleration gap. The interpretation of the soft X-ray emission is complicated by the presence of the thermal surface flux, which itself may be pulsed if the surface temperature is nonuniform. The radio emission comes from low altitudes and, being more tightly beamed, shows narrower pulses which cover a smaller portion of the sky. The exception to this picture is the Crab, whose nonthermal accelerators seem to be strong enough to keep pair production rates and particle densities high enough to ensure that all emissions (radio to $\gamma$-ray) are dominated by the outer gap zone.

This interpretation has some encouraging successes but is not unique. In particular, Harding and colleagues (e.g., Daugherty \& Harding 1996) have described a polar cap model based on aligned rotators that can produce high-energy hollow-cone emission of the observed width. The highest $\mathrm{S} / \mathrm{N}$ light curves also suggest components in addition to the dominant double pulse which may require other beams in the magnetosphere or perturbations from the simple, swept-back dipole geometry.

\section{Continuum Spectral Features}

Light curves help locate the position of the radiation zones, but spectral energy distributions (SEDs) are the key to identifying the radiation processes. Thompson (1998) has compiled a useful set of broadband SEDs for the young pulsars. The most striking feature of these $\nu F_{\nu}$ diagrams is the peaking of the photon power at $>100 \mathrm{MeV}$, with a rollover of the spectrum at several GeV. This characteristic energy clearly defines the radiation-reaction limit process for the primary magnetosphere particles. Most recent models (e.g., Daugherty \& Harding 1996; Romani 1996; Zhang \& Cheng 1997; Hirotani \& Shibata 1999) conclude that the particles are curvature radiation limited. The original Cheng, Ho, \& Ruderman (1986) picture and the polar cap picture of Dermer \& Sturner (1994) focused on ICS limited particles, but these schemes require either improbably large fluxes of target soft photons or surprisingly low primary $\Gamma$ factors.

There are important differences in current schemes, though. Polar cap models make the required pairs in a $\gamma-B$ cascade, and the $\mathrm{GeV}$ cutoff, identified with the absorption wall for this process, should be quite steep (Daugherty \& 


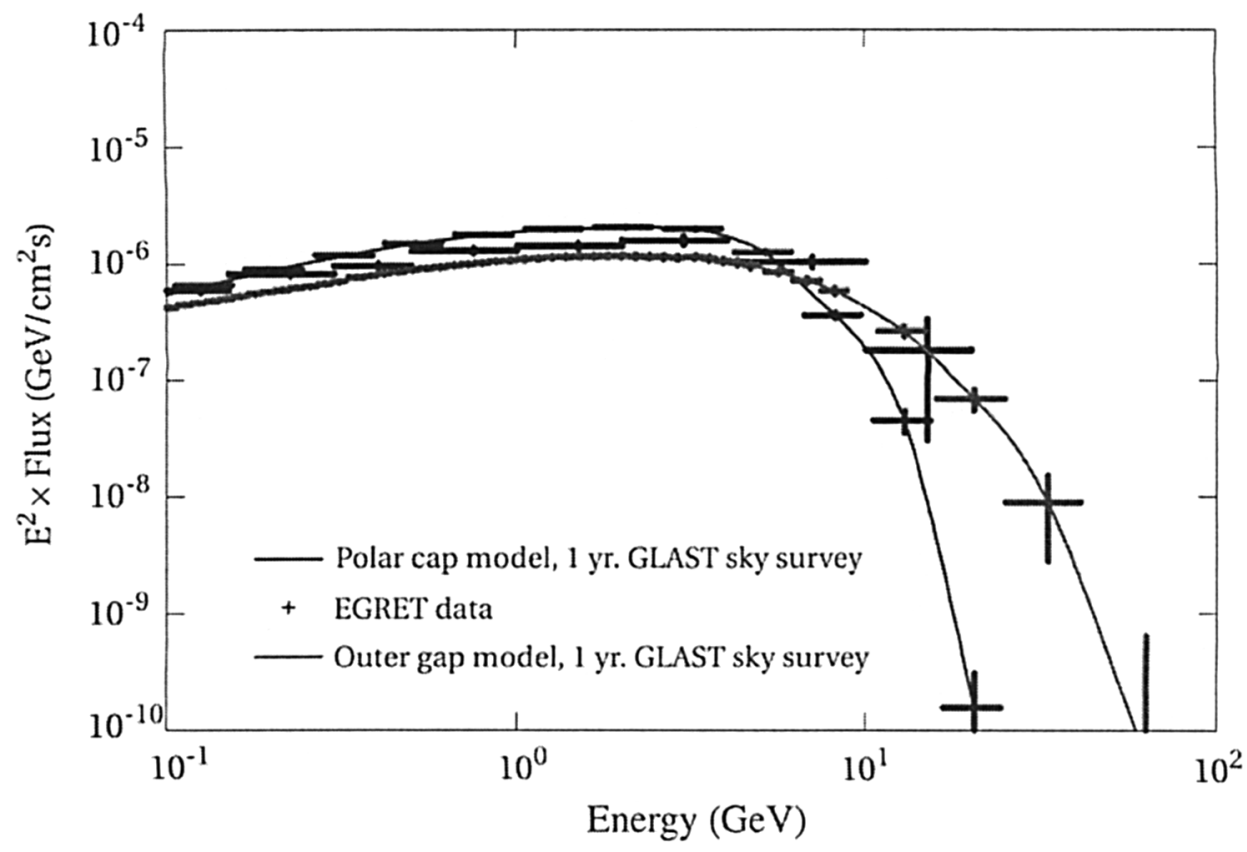

Figure 2. A comparison of the EGRET data for Vela (isolated error flags) with predictions of an outer gap model (upper curve) and a polar cap model (lower curve). Simulated GLAST data are shown by the error flags on these two curves (Thompson et al. 1999).

Harding 1996). In contrast, pairs in outer magnetosphere scenarios must be produced via $\gamma-\gamma$ collisions, and the $\mathrm{GeV}$ cutoff is identified directly with the more gradual rollover of the primary curvature spectrum for $\Gamma \sim 10^{7.5} e^{ \pm}$in the outer magnetosphere. Existing data (Figure 2) provides some check of these interpretations, but a definitive answer, even for the brighter $\gamma$-ray pulsars, awaits a next generation GeV observatory, such as GLAST.

The next prominent, nonthermal feature is the break from the $\gamma$-ray component to a steeper power law at hard X-ray energies, continuing through to the optical nonthermal fluxes. This component is interrupted in the $10^{4}-10^{6} \mathrm{y}$ pulsars by a Planck-like excess from the neutron star surface which dominates at $\sim 10-10^{3} \mathrm{eV}$. The power law spectrum is widely interpreted as synchrotron emission, and the upper limit of this component at $\sim \mathrm{MeV}$ energies is a diagnostic of the initial energy and pitch angle $\Psi$ of the cooling $e^{ \pm}$. For example, at the null charge surface $\left(r_{i} \sim 0.1 r_{L C}\right)$, we expect the synchrotron component to peak at $E \sim 0.5 B_{12}^{5 / 2}(P / 0.1 s)^{-13 / 2}\left(r_{i} / 0.1 r_{L C}\right)^{-2} \mathrm{MeV}$ for $\Psi \sim r_{i} / r_{L C}$, induced by aberration. It will be particularly valuable to map the slope and upper cutoff of this $\mathrm{MeV}$ component as it varies with pulse phase. It is a direct probe of the properties of the synchrotron electrons at birth and identifies the pair generation process and the target field. This difficult energy range may be best probed by the next generation "Advanced Compton Telescope". 
One continuum component of particular interest is, as yet, unobserved. This is the pulsed $\mathrm{TeV}$ flux which must be produced through Compton upscattering by the $\Gamma>10^{7}$ primaries. It is likely that this flux is substantially below present upper limits (Hirotani 2000), but since its amplitude is set by the poorly constrained soft target flux and its unknown angular distribution, upper limits continue to be a valuable check on acceleration models. Detection of $\mathrm{TeV}$ pulsations would directly probe the radiation-reaction limited energy and provide key information on acceleration potentials and gap geometries.

Finally, we turn to the soft (IR-UV) end of the synchrotron spectrum. With the exception of the Crab-like pulsars, this emission is faint, typically $m_{V}>25$ even for the nearest young objects. Nevertheless, several factors make the study of this continuum valuable. While energetically unimportant, as seen on $\log \left(\nu F_{\nu}\right)$ plots, this flux dominates the nonthermal photon number flux and provides a peak in the $\log \left(\mathrm{d} N_{\gamma} / \mathrm{d} E_{\gamma}\right)$ plots. So, these photons dominate the Compton target emission, for example. Also, relatively large number fluxes make sensitive, phase-resolved measurements and pulse-to-pulse variability studies possible (at least in principle). With a large energy lever arm, the optical continuum flux can be compared with the hard X-ray component to study the $e^{ \pm}$synchrotron evolution. Finally, with modern detectors in the optical/nearIR, polarization is measurable in several cases, which provides a unique window on the magnetic field geometry.

\subsection{Lines and Edges}

Spectral indices and cutoffs allow us to trace the main processes in the emission zone, but to obtain detailed measurements of the physical parameters line and edge features would be especially valuable. Such study of pulsar magnetospheres is very much in its infancy, but the next generation observational technology should provide important advances in this area. Given the presence of an $e^{ \pm}$flow in the magnetosphere and continuous pair creation, annihilation lines should be present. Discussing the tentative FIGARO detection of pair annihilation from the Crab at a few $R_{\mathrm{NS}}$, Zhu \& Ruderman (1997) and Wang (1998) describe a "cyclotron resonant blanket" in which the pair production/annihilation rate may exceed the corotation charge density flow by a large factor, up to $10^{6} \dot{n}_{\mathrm{GJ}}$. Detailed measurement of the phase and energy structure of this annihilation line promises to provide an excellent tracer of magnetosphere plasma generation; the INTEGRAL may make good progress in this area.

The corotating pair plasma may also be detected by its effect on the underlying thermal emission. For example, Rajagopal \& Romani (1997) and Braje $\&$ Romani (2000) have described light curve perturbations caused by cyclotron resonant scattering of the surface emission, where the minimum expected corotation charge density should produce detectable optical depths for young pulsars in soft X-rays $\left[\tau \approx 0.01 n_{\mathrm{GJ}}(0.1 s / P)\right]$. Even stronger scattering can be present for low- $B$ pulsars in the optical/UV.

The IR-optical-UV synchrotron emission also apparently contains lines and edges. Figure 3 shows a plot of some recent HST and ground-based photometry of several young, spin-powered pulsars (Romani et al. 1999; Nasuti et al. 1997; Pavlov, Welty, \& Cordova 1997; Mignani, Caraveo, \& Bignami 1998). For comparison, two component models are plotted: a synchrotron power-law with 


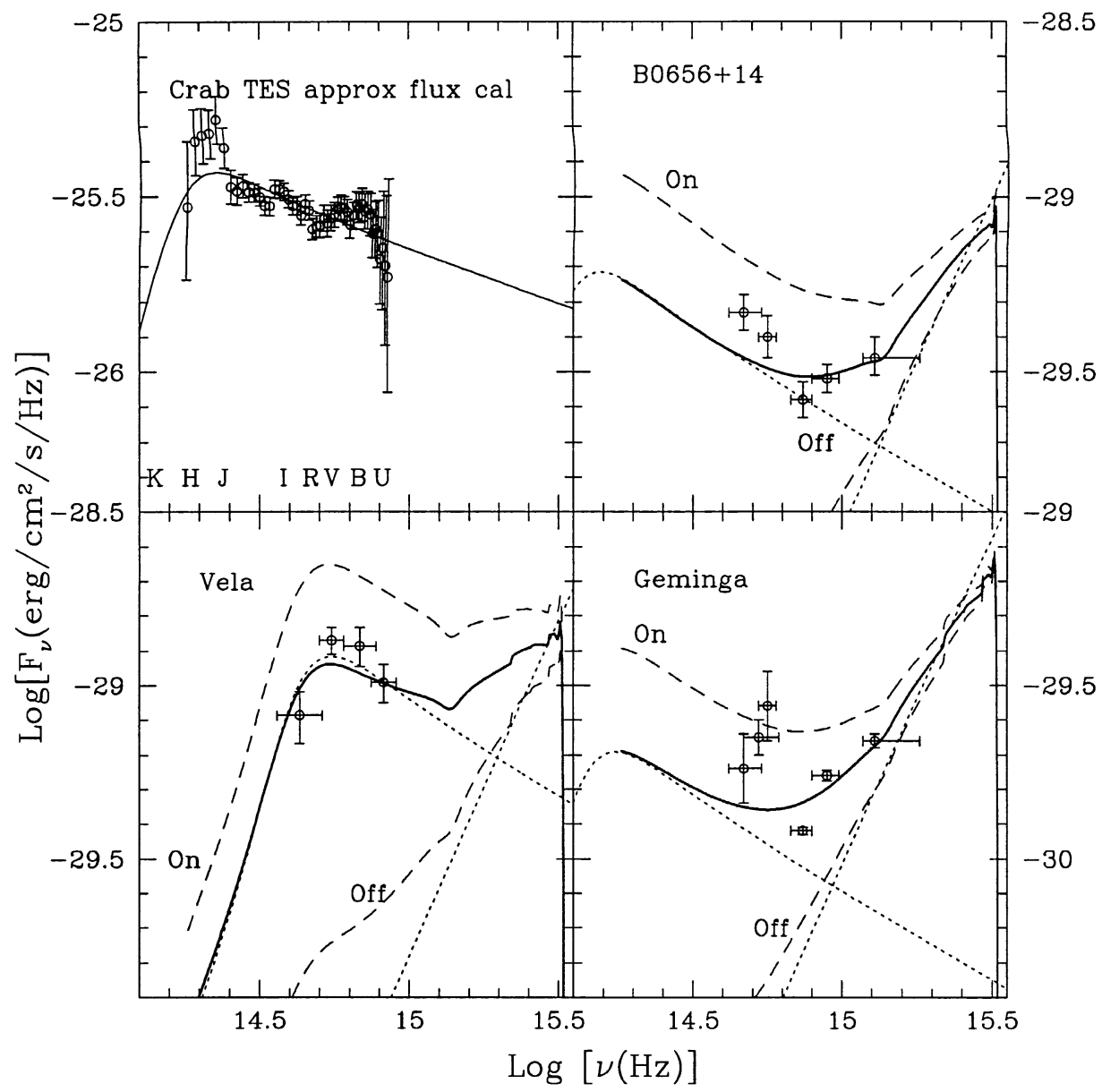

Figure 3. Near IR - UV spectral energy distributions of young pulsars.

a simple self-absorption cutoff and the Rayleigh-Jeans-like tail of a thermal surface spectrum (incorporating magnetized Fe atmosphere, Rajagopal, Romani, \& Miller 1997). The synchrotron component extrapolates to the hard X-ray $(E>2 \mathrm{keV}$ ) measurements or upper limits with an $\alpha \approx 0.5$ power law (cf. Wang et al. 1998). This component is highly pulsed, and so, in addition to the phase-averaged solid line, dashed lines show typical on- and off-pulse amplitudes for the summed spectrum. New HST NICMOS data confirm the plotted rise of Geminga and PSR B0656+14 in the near IR (Pavlov, private comm.). Because self-absorption is present first at the pulse peaks, the IR turnover will be less steep than the single zone absorption shown here.

At least for PSR B0656+14 and Geminga, the phase-averaged photometry suggests high equivalent-width departures from a simple power law. A phaseaveraged spectrum of Geminga (Martin, Halpern, \& Shiminovich 1998) supports the existence of optical features, as well. It is interesting that both near surface and outer magnetosphere scenarios for high-energy emission can be used 
to interpret such optical/UV features. For example, Jacchia et al. (1999) have interpreted the Geminga colors as ion cyclotron features at the star surface, where, for ${ }^{1} \mathrm{H}, E \sim 5 \mathrm{eV} B_{\perp 12}$. The fits suggest a magnetic pole temperature of $\sim 10^{8} \mathrm{~K}$ and a field strength of $B \sim 4 \times 10^{11} \mathrm{G}$ (hydrogen) or $B \sim 8 \times 10^{11} \mathrm{G}$ (helium). Since the implied field values are low and the cap temperature is rather high for this pulsar, and since the rising IR flux argues against the R-J interpretation, this particular scenario is unlikely to be correct, but the possibility of surface features in this range should not be discounted. Line features at $\sim \mathrm{eV}$ energies are also natural in the outer magnetosphere picture since, at the null charge surface, fields are $\sim 10^{4}$ times lower than at the star, and the $e^{ \pm}$ fundamental has $E \sim 0.8 \mathrm{eV} B_{12}\left(r_{0.05} P_{-1}\right)^{-3}$, if the null charge surface is at $1 / 20$ of the light cylinder radius. In this interpretation, the observed features may be produced by outer gap beams crossing cold plasma, which cyclotron-scatters the gap radiation.

In the outer magnetosphere, we can also expect spectral breaks. For example, the Crab optical spectrum flattens at $\sim 2 \mathrm{eV}$, which may be related to the low-energy cutoff of the synchrotron particle populations in the outer magnetosphere. There is good evidence from an even steeper break to the near IR that the main pulse, at least, is self-absorbed (Penny 1982; Middleditch, Pennypacker, \& Burns 1983; Romani et al. 1999). Of course, given the variation of $B, \Psi$, and $N_{e}$ along the instantaneous line-of-sight, one expects all of these features to display strong phase variations. Indeed, for the Crab, broadband phase-resolved colors indicate continuum slope variations across the visible pulse (Sanwal, Robinson, \& Stiening 1998). The presence of any line feature in Geminga is likely due to the optical pulse being dominated by a relatively narrow phase window (Shearer et al. 1998), but phase-resolved data should reveal changes even through this pulse.

This is both an opportunity and a challenge. The presence of phase-varying spectral features, tracing directly the $B$ field and plasma properties as a function of position in the pulse emission zone, offers the possibility of a real tomographic map of the pulsar acceleration gap and its radiation zone. However, the typically faint fluxes of even nearby pulsars $\left(F_{\nu} \sim 10^{-30} \mathrm{erg} / \mathrm{cm}^{2} \mathrm{~s} \mathrm{~Hz}\right)$ make the extraction of the required phase-resolved spectra particularly challenging.

Recently, cryogenic detector technology has made encouraging progress. Superconducting detectors are now being developed (Peacock et al. 1996; Cabrera et al. 1998) that provide energy-resolved photon counting with good efficiencies covering the near IR-optical-UV range. These devices have already been applied to test observations of the Crab pulsar (Romani et al. 1999; Perryman et al. 1999). The combination of sensitivity, high time resolution, and good energy coverage should make sensitive measurements of the nonthermal emission and its spectral features possible. When coupled with true 3-D models, accounting for the particular field line bundles occupied by the radiating plasma, and for strong beaming of the emission itself, these phase-resolved measurements, especially in the optical/UV, should provide the diagnostics needed to advance solutions of a variety of pulsar emission puzzles. 


\section{References}

Becker, W. 2000, these proceedings

Braje, T. M., \& Romani, R. W. 2000, ApJ, submitted

Cabrera, B., et al. 1998, Appl. Phys. Lett., 73, 735

Cheng, K. S, Ho, C., \& Ruderman, M. 1986, ApJ, 300, 522

Daugherty, J. K., \& Harding, A. K. 1996, ApJ, 458, 278

Dermer, C. D., \& Sturner, S. J. 1994, ApJ, 420, L75

Hirotani, K. 2000, ApJ, submitted

Hirotani, K., \& Shibata, S. 1999, MNRAS, 308, 54

Jacchia, A. et al. 1999, A\&A, 347, 494

Lyne, A. G., \& Manchester, R. N. 1988, MNRAS, 234, 477

Lyutikov, M., Blandford, R., \& Machabeli, G. 2000, these proceedings

Martin, C., Halpern, J. P., \& Shiminovich, D. 1998, ApJ, 494, L211

Middleditch, J., Pennypacker, C., \& Burns, M. S. 1983, ApJ, 273, 261

Mignani, R., Caraveo, P. A., \& Bignami, G. F. 1998, Adv. Space Res., 21197

Morini, M. 1983, MNRAS, 202, 495

Pacini, F. 2000, these proceedings

Pavlov, G. G., Welty, A., \& Cordova, F. A. 1997, ApJ, 489, L75

Pavlov, G. G., \& Zavlin, V. E. 2000, these proceedings

Peacock, A. et al. 1996, Nature, 381, 135

Penny, A. J. 1982, MNRAS, 198, 773

Perryman, M. A. C., et al. 1999, A\&A, 346, L30

Radhakrishnan, V., \& Cooke, D. J. 1969, Astrophys. Lett., 3, 225

Rajagopal, M., \& Romani, R. W. 1997, ApJ, 491, 296

Rajagopal, M., Romani, R. W., \& Miller, M. C. 1997, ApJ, 479, 437

Romani, R. W. 1996, ApJ, 470, 469

Romani, R. W., et al. 1999, ApJ, 521, L153

Romani, R. W., \& Yadigaroglu, I.-A. 1995, ApJ, 438, 314

Sanwal, D., Robinson, E. L., \& Stiening, R. F. 1998, BAAS, 30, 1420; also Sanwal, D., Ph.D. Thesis, in preparation

Shearer, A., et al. 1998, A\&A, 335, L21

Thompson, D. J. 1998, in Neutron Stars and Pulsars, eds. N. Shibazaki, N. Kawai, S. Shibata, \& T. Kifune (Tokyo: Universal Academy), 273

Thompson, D. J, Harding, A. K., Mattox, J. R., \& Romani, R. W. 1999, unpublished

Wang, F. Y.-H. 1998, in Neutron Stars and Pulsars, eds. N. Shibazaki, N. Kawai,

S. Shibata, \& T. Kifune (Tokyo: Universal Academy), 351

Zhang, L., \& Cheng, K. S. 1997, MNRAS, 487, 370

Zhu, T., \& Ruderman, M. 1997, ApJ, 478, 701 